\title{
Experimental Therapeutics in Treatment-Resistant Major Depressive Disorder
}

This article was published in the following Dove Press journal:

Journal of Experimental Pharmacology

\author{
Mandeep Kaur' \\ Marsal Sanches ${ }^{2}$ \\ 'Department of Behavioral Medicine, \\ Cone Health, Greensboro, NC, USA; \\ ${ }^{2}$ UT Health Center of Excellence on \\ Mood Disorders, Faillace Department of \\ Psychiatry \& Behavioral Sciences, \\ McGovern Medical School, Houston, \\ TX, USA
}

\begin{abstract}
Treatment-Resistant Depression (TRD) patients remain a challenging subdivision of patients with Major Depressive Disorder (MDD) in day to day clinical practice. As with any diagnostic condition, comprehensive evaluation, exclusion of other psychiatric conditions, assessment of co-morbid medical and psychiatric illnesses and psychosocial stressors are the keys to appropriate diagnosis and subsequent management. There are various management options available for the treatment of MDD, however, about $30 \%$ of the patients fail to achieve full remission of symptoms despite multiple trials and belong to this sub-category of MDD. This article brings forth discussion of other non-conventional medication and non-medication treatment alternatives that merit exploration of their efficacy in TRD. Many of the proposed novel medications and other treatment modalities such as Deep Brain Stimulation, exercise, yoga are already used for other medical and psychiatric disorders and have some evidence suggesting their potential benefits in TRD in conjunction with conventional medications or even as monotherapy. Nevertheless, more research is needed in this direction to establish effectiveness.
\end{abstract}

Keywords: major depressive disorder, treatment-resistant depression, anti-depressants, pharmacology, deep brain stimulation, HPA axis, psychedelic agents

\section{Introduction}

Globally, more than 264 million people of all ages suffer from depression. ${ }^{1}$ In 2017 , an estimated 17.3 million adults in the United States had at least one major depressive episode, representing $7.1 \%$ of all adults. ${ }^{2}$ Major Depressive Disorder (MDD) is defined as a period of at least two weeks when a person experiences a depressed mood or loss of interest or pleasure in daily activities, and has majority of specified symptoms, such as problems with sleep, eating, energy, concentration, or self-worth. ${ }^{3}$ The recurring and debilitating nature of the illness causes significant impairment of individual functioning. Depression is a leading cause of suicide and is also associated with different medical conditions, such as obesity, diabetes, and stroke, as well as a greater risk for Alzheimer's disease and sudden cardiac death. ${ }^{4}$ Depression is moderately heritable with approximate $35 \%$ of the risk for MDD associated with genetic predisposition, ${ }^{5}$ and is highly influenced by adverse life experiences. $^{6}$

Significant advances have been reached in understanding the pathophysiology of depression, and various treatment modalities now exist, including antidepressant medications, different forms of psychotherapy, and brain stimulation techniques. The treatment plan for depression typically involves trial and error procedures
Correspondence: Mandeep Kaur General, Child and Adolescent Psychiatrist Department of Behavioral Medicine, Cone Behavioral Health Hospital, 700 Walter Reed Drive, Greensboro, North Carolina, 27410, USA Tel +I (7I3) 540-2469

Email mandy.drmandeep@gmail.com
Journal of Experimental Pharmacology 2021:13 181-196

| 8 |

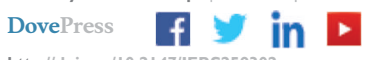

(c) (i) (5) 2021 Kaur and Sanches. This work is published and licensed by Dove Medical Press Limited. The full terms of this license are available at https://www.dovepress.com/ BY NC terms.php and incorporate the Creative Commons Attribution - Non Commercial (unported, v3.0) License (http://creativecommons.org/licenses/bync/3.0). By accessing the work you hereby accept the Terms. Non-commercial uses of the work are permitted without any further permission from Dove Medical Press Limited, provided the work is properly attributed. For permission for commercial use of this work, please see paragraphs 4.2 and 5 of our Terms (https://www.dovepress.com/terms.php). 
attempting to match the patients with the treatment strategy that best works for them. Despite the advances in the field, a subset of depressive disorders remains a therapeutic challenge; these cases are often grouped under the term treatment-resistant major depressive disorder. There are several different definitions of treatmentresistant depression (TRD); for example, the term may refer to failure to respond to a treatment intervention within a depressive episode ${ }^{7}$ or to a previously effective treatment in a subsequent episode. ${ }^{8}$ A popular definition states that TRD corresponds to the depression that persists after two adequate trials of treatment modalities according to the evidence-based sequence that prescriber considers to be appropriate for the patient. ${ }^{9}$ Some experts point out the need to focus on understanding the nature and causes of persistence of depression. ${ }^{10}$ Issues like whether or not partial response should be deemed as non-resistance as opposed to resistance and should treatment be measured completely independently from history and life context are imperative to consider. ${ }^{10}$ Pseudoresistance is another concern since substantial evidence indicates that large numbers of depressed patients are undertreated, resulting in prolonged episodes. ${ }^{11}$ It is suggested that the adequacy of antidepressant treatment trials be defined in terms of thresholds for the dosage and duration of medication, adherence, and clinical outcome. ${ }^{11}$ Difficult-to-treat depression is another concept proposed, as it is more accepting of the fact that the treatment aims for some depressed patients may shift to optimal symptom control rather than remission. ${ }^{12}$ The creators of this concept point out that TRD does not designate a biologically homogeneous group demarcated by a clear line. ${ }^{12}$

The Sequenced Treatment Alternatives to Treat Depression $\left(\mathrm{STAR}^{*} \mathrm{D}\right)$ trial, one of the largest studies in depression, was designed to give guidance in selecting the best next-step treatment for the patients who failed to achieve adequate relief from the initially used medication in the given 4 -step process. About 33\% entered remission after one treatment step, $50 \%$ after two steps, $60 \%$ after three steps, and $70 \%$ after four steps. ${ }^{13}$ Taking the findings of this landmark study into account, an estimated 30\% patients would fall under the treatment-resistant category. ${ }^{13}$

The underlying pathophysiological basis leading to certain patients becoming treatment-resistant is unknown. A vast body of research suggests that early childhood adverse effects like abuse and trauma predict poorer response to antidepressant treatment in later life. ${ }^{14}$
Neuroimaging studies have revealed deviations in the functional circuit connectivity in treatment-resistant patients, with the subcallosal cingulate cortex (SCC) corresponding to a core component of MDD pathophysiology. ${ }^{15}$ Greater functional connectivity between the SCC and the default mode network has been reported among patients with TRD. ${ }^{16}$ Treatment-resistance can take place in patients previously responsive to treatment or present as a subset of patients with depression who display a progressive, deteriorating illness course over time. ${ }^{17}$ Another possible underlying mechanism is the presence of interpersonal relationship disruptions known as "dyadic discord", which has been associated with higher depressive symptoms and reduced likelihood of remission in spite of treatment. ${ }^{18}$

In the present review, we provide a critical analysis of novel treatments for TRD, in light of available evidence. In addition to novel pharmacological treatments, we also discuss the potential role of experimental neuromodulation techniques and other non-pharmacological therapeutic approaches currently under investigation for the treatment of depression.

\section{Methods}

The database PubMed (2010-2020) was searched, using the MeSH terms "treatment resistant depression", "treatment refractory depression", "depression", "antidepressant therapy". Furthermore, a manual search of bibliographical cross-referencing was conducted. The papers identified were then manually screened for original articles and reviews focusing on resistant depression. Additionally, the online database "clinicaltrials.gov" was searched for trials targeting patients with refractory or resistant depression, and the results obtained were manually screened as well.

\section{Medications Targeting the Hypothalamic-Pituitary-Adrenal (HPA) Axis}

Stressful life events are directly associated with dysfunction in the hypothalamic-pituitary-adrenal (HPA) axis in depression as evidenced by elevated cortisol levels, reduced neurogenesis, decreased synaptic plasticity and hippocampal atrophy. ${ }^{19}$ HPA axis abnormalities have been linked to both childhood trauma and depression, but the underlying mechanisms are poorly understood. ${ }^{20}$ A significant proportion of depressed patients exhibit 
hyperactivation of the HPA axis resulting in increased secretion of the stress hormone cortisol as well as elevated levels of inflammatory biomarkers. ${ }^{21}$ It has been suggested that such disturbances are due to the dysfunction of glucocorticoid receptors (GR), and consequent impairment to mediate HPA axis negative feedback by cortisol which promotes anti-inflammatory response. Attenuated glucocorticoid responsiveness or glucocorticoid resistance has been consistently observed in MDD patients presenting coexistence of hypercortisolemia and inflammation. ${ }^{20,21}$ Based on this evidence, HPA axis has been regarded as a possible target for novel therapeutic agents in TRD.

\section{Mifepristone}

Mifepristone is a competitive GR antagonist that has been investigated for the treatment of psychotic depression, based on the observation that patients with MDD with psychotic symptoms seem to have particularly significant cortisol dysregulation. ${ }^{22}$ A double-blind, multi-site randomized clinical trial (RCT) included 292 patients with a diagnosis of MDD with psychotic features who underwent daily administration of oral mifepristone $1200 \mathrm{mg}$ or placebo for days 1 to 7 of the 56-day trial, followed by treatment with a single FDA-approved antidepressant on days 8 to $56 .{ }^{23}$ Patients who attained a mifepristone plasma level of $1637 \mathrm{ng} / \mathrm{mL}$ or greater demonstrated significant reductions in psychotic symptoms compared with patients who received placebo starting on day 28. No significant improvements were observed in the low-mifepristone group versus the placebo group. In another trial, 433 patients with psychotic depression were randomly assigned to receive 7 days of either mifepristone at doses $300 \mathrm{mg}, 600 \mathrm{mg}, 1200 \mathrm{mg}$ or placebo. ${ }^{24}$ It was noted that patients with trough mifepristone plasma concentrations greater than $1660 \mathrm{ng} / \mathrm{mL}$ were significantly more likely to have a rapid and sustained reduction in psychotic symptoms than those who received placebo. The replication of this association between plasma concentration and clinical response indicates that mifepristone at sufficient plasma levels may be effective in reducing the psychotic symptoms in patients with psychotic depression. The good tolerability of mifepristone in the trials suggest its role and potential use in TRD deserves further exploration.

\section{Serum and Glucocorticoid-Inducible Kinase I (SGKI)}

Neurogenesis seems to play an important role in antidepressant treatment response and HPA axis regulation. ${ }^{25}$ SGK1 is a serine/threonine kinase GR target gene implicated in cellular stress response and neuronal function. SGK1 is a key enzyme involved in the downstream mechanisms by which glucocorticoids reduce neurogenesis and in the upstream potentiation and maintenance of GR function, even after glucocorticoid withdrawal. SGK1 mediates glucocorticoid effects on brain function; for example, acute stress enhances neuronal excitability and working memory in the prefrontal cortex of rodents via glucocorticoidinduced activation of SGK1. ${ }^{25}$ Additionally, SGK1 mRNA has been found to be increased in the blood of drug-free depressed patients and in the hippocampus of rats after chronic mild stress. ${ }^{26}$ In light of the findings, SGK1 may represent an antidepressant treatment strategy to counteract stress-induced impairments in neurogenesis and associated biological abnormalities in depression and TRD.

\section{Metyrapone}

Metyrapone is an inhibitor of the enzyme 11- $\beta$ hydroxylase that catalyzes the conversion of 11-deoxycortisol to cortisol. A number of studies have utilized metyrapone as an augmenting agent for antidepressants. One 2-month long open-label study using metyrapone in 8 patients with TRD was reported. ${ }^{27}$ Treatment included use of metyrapone alone or concomitant use of other steroid synthesis inhibitors (aminoglutethimide, ketoconazole). Remission of symptoms was noted for 6 of these patients. ${ }^{27}$ A single-blind crossover study of 2 weeks' treatment with metyrapone combined with replacement therapy with hydrocortisone in 8 inpatients with depression found significant reduction in depression symptoms in the treatment group compared to placebo. ${ }^{28}$ The most robust evidence for the use of metyrapone in depression comes from a placebo-controlled RCT. ${ }^{29}$ In total, 63 inpatients with depression received augmentation of nefazodone or fluvoxamine for 3 weeks with placebo or $1 \mathrm{~g}$ of metyrapone once daily. The group treated with metyrapone showed a significantly greater improvement compared with the placebo group. Unlike some of the previous studies, this study used a standard dose of metyrapone (rather than adjusting the dose according to cortisol levels). However, the exact mechanism of the antidepressant effect of metyrapone is unclear. The absence of a significant reduction of cortisol in this study argues against a simple reduction in plasma cortisol being the mechanism of action. Metyrapone was well-tolerated. 


\section{Fludrocortisone}

Fludrocortisone is a mineralocorticoid receptor (MR) agonist. Under normal circumstances, endogenous glucocorticoids bind to the GRs and the MRs in the brain, and act as negative regulators of HPA axis activity. In the brain, MRs have ten times the affinity of GRs to glucocorticoids and are vital to controlling the HPA response to stress. ${ }^{30}$ Animal studies have reported that antidepressants (fluoxetine) increase central mineralocorticoid receptors. ${ }^{31}$ In one RCT of 64 patients, combining the MR agonist fludrocortisone with the antidepressant escitalopram vs MR antagonist spironolactone resulted in decreased plasma cortisol levels and improved response time. ${ }^{32}$ However, this study did not assess HPA axis feedback inhibition and did not study any sub-types of depression. Another study looked at the role of fludrocortisone in differentially inhibiting the HPA axis of 14 psychotic major depression subjects, 16 non-psychotic major depression subjects, and 19 healthy control subjects. ${ }^{33}$ This study demonstrated that individuals with psychotic major depression, compared to healthy control subjects, had diminished feedback inhibition of the HPA axis in response to fludrocortisone. Notably, fludrocortisone at higher doses may not be a practical long-term treatment due to side effects such as hypertension.

\section{Testosterone (in Females)}

With the exception of testosterone replacement for hypogonadal men, evidence for testosterone's antidepressant role is modest. In 2019, intravenous brexanolone was FDA approved for postpartum depression making it the first neuro-steroid medication approved. ${ }^{34} \mathrm{~A}$ recent double-blind placebo-controlled RCT looked at role of adjunctive testosterone cream in 101 women ages $21-70$ with TRD over a period of 8 weeks. ${ }^{35}$ Primary outcome was depression severity and secondary outcomes were fatigue, sexual function, and safety measures. A functional MRI sub-study examined the effects on activity in anterior cingulate cortex (ACC) and primary outcome was change in ACC activity. MADRS scores decreased in both study arms from baseline to week with no significant difference between groups. Improvement in fatigue and sexual function did not differ between groups, nor did side effects. fMRI results showed a relationship between ACC activation and androgen levels before treatment but no difference in ACC activation with testosterone compared with placebo. The authors noted that placebo response rate in this trial was high (49\%) which may have accounted for lack of observed treatment effect.

\section{Medication Targeting the Melatonergic System}

Melatonin modulates circadian rhythms through signaling pathways coupled to the melatonin receptors MT1 and MT2. Both the receptors are involved in mediating anhedonic- and anxiety-like behaviors. ${ }^{36}$ In rodents, melatonin stimulates all stages of neuroplasticity which is an important process that follows a circadian rhythm for maintaining appropriate mental health. ${ }^{37}$ Melatonin is also known to stimulate dendritogenesis and synaptogenesis in the hippocampus. ${ }^{37}$

\section{Agomelatine}

Agomelatine is both a melatonin agonist and a selective serotonin antagonist. Agomelatine induces resynchronization of circadian rhythms and enhances the levels of dopamine and noradrenaline in the frontal cortex. ${ }^{38}$ A number of case reports have looked at combinations of various antidepressants (escitalopram, venlafaxine, clomipramine) with agomelatine resulting in improved outcomes in depression as well as obsessive compulsive disorder. ${ }^{38}$ One case report depicted successful remission of TRD by combining moclobemide with agomelatine. ${ }^{39}$ Another case report showed the combination of bupropion with agomelatine as a successful strategy for TRD. ${ }^{40}$ One RCT involving a 12-week treatment period and a double-blind extension for 12 weeks compared agomelatine and escitalopram for efficacy, subjective sleep and tolerability. ${ }^{41}$ The findings suggested that both agomelatine (164 patients) and escitalopram (160 patients) improved depressive symptoms with agomelatine being better-tolerated than escitalopram. Additionally, agomelatine displayed longterm benefits on sleep-wake quality and emotional experiences like lower emotional blunting over escitalopram in the management of depression.

A bigger 12-week open label non-interventional study (VIVALDI study), looked at 3317 patients and reported that agomelatine improved depressive symptoms and daytime functioning as a monotherapy in 987 patients as well as an add-on therapy to the drugs used for the treatment of a depressive episode in 856 pretreated patients; in addition to 697 patients who switched to agomelatine under routine clinical practice. ${ }^{42}$ Agomelatine was effective and well tolerated in combination with other antidepressants. 
Monitoring of hepatic function is recommended due to several reports of hepatotoxicity. Recent availability of intranasal agomelatine may help to circumvent this adverse effect. ${ }^{43}$

\section{Medications Targeting the Glutamatergic System}

Ketamine is an anesthetic and analgesic agent that affects fast excitatory glutamate transmission, increases BDNF release, and stimulates synaptogenesis. ${ }^{44}$ The pharmacodynamics of ketamine and esketamine are well demonstrated with clinical antidepressant effects appearing in minutes to hours post administration in line with glutamate hypothesis of major depression. ${ }^{44}$ Studies show that single doses of ketamine should be considered as an option in TRD patients with/without suicidal thoughts; even though it may not lead to remission, ketamine seems to improve quality of life by reducing depressive symptomatology. ${ }^{45}$ Studies also indicate favorable safety and tolerability profile of ketamine in TRD including co-morbid cardiovascular disease. ${ }^{44,45}$ One multi-center RCT evaluated efficacy of twice- and thrice-weekly intravenous administration of ketamine in sustaining initial antidepressant effects in patients with TRD. ${ }^{46}$ Both treatment regimens resulted in improvement in depressive symptoms in 67 of 68 randomized patients and antidepressant efficacy was maintained over 15 days.

Esketamine, the S-enantiomer of racemic ketamine, which has a higher affinity for the N-methyl-D-aspartate (NMDA) receptor, has been approved in the US as a nasal spray formulation for TRD. ${ }^{47}$ Meta-analysis of RCTs examining the effectiveness, tolerability, and safety of intranasal esketamine in treating MDD indicated that intranasal esketamine appears to have an ultra-rapid antidepressant effect, lasting for at least 28 days. ${ }^{48}$ Another metaanalysis of several RCTs concluded that adjunctive intranasal esketamine appears to be an effective treatment strategy for patients with MDD who are either treatmentresistant or acutely suicidal. ${ }^{49}$ The European Medicines Agency (EMA) approved esketamine nasal spray, in combination with a selective serotonin reuptake inhibitor or a serotonin-norepinephrine reuptake inhibitor, for adults with TRD. ${ }^{50}$ These finding have opened up the avenue of use of other agents targeting glutamatergic system. ${ }^{51}$ Some of the NMDA receptor modulators that are under investigation for treatment of TRD are discussed below.

\section{Rapastinel}

Rapastinel acts as a weak partial agonist of NMDA receptor. A pilot Phase II RCT showed that a single intravenous dose of rapastinel reduced depressive symptoms within 2 hours, and this effect was maintained for around 7 days. ${ }^{52}$ Further trials are in early phases.

\section{AV- I0I}

The kynurenine pathway plays a critical role in generating cellular energy in the form of nicotinamide adenine dinucleotide. ${ }^{53}$ In this pathway, tryptophan is converted into several bioactive molecules including serotonin while majority of it is converted into kynurenine and its breakdown products. Through a series of steps kynurenine is converted into quinolinic acid, which has NMDA receptor agonist properties and kynurenic acid, which is an NMDA receptor antagonist. A hypothesis suggests that the competing actions of quinolinic acid and kynurenic acid at the NMDA receptor may play role in inflammation and glutamate models of depression. ${ }^{53}$ NMDA receptor activity requires binding of glycine or D-serine to coagonist glycine site. ${ }^{54} \mathrm{AV}-101$ or 4-chlorokynurenine is a prodrug that is rapidly converted in vivo to its active metabolite 7-Choloro-kynurenic acid, which works as an antagonist at the NMDA glycine site thus exerting its NMDA receptor antagonistic property. ${ }^{54}$ In a small crossover placebo-controlled trial looking at its efficacy in TRD, it was noted that monotherapy with 4-Chlorokynurenine exerted no antidepressant effects at the doses and treatment duration studied. ${ }^{54}$

\section{CERC-30I}

CERC-301 is a highly selective, orally bioavailable, NMDA receptor antagonist. A small RCT suggested antidepressant efficacy for CERC-301 in TRD. ${ }^{55}$

\section{AVP-923 (Nuedexta)}

Commonly known by its brand name nuedexta, this medication was the first FDA approved medication for pseudobulbar affect. AVP-923 is a fixed combination of dextromethorphan and quinidine. Dextromethorphan is a noncompetitive NMDA receptor antagonist and $\sigma 1$-receptor agonist and quinidine is a CYP2D6 enzyme inhibitor, which serves to increase plasma concentrations of dextromethorphan and prolong its plasma half-life. A small one-arm Phase II study designed to evaluate the efficacy, safety, and tolerability of AVP-923 in patients with 
TRD looked at the primary outcome measure of change in MADRS total score from baseline to week $10 .^{51}$ The study was recently completed, but results are not yet available.

\section{AVP-786 \\ (Deuterated-Dextromethorphan/ Quinidine Combination)}

This is a combination of deuterated-dextromethorphan and an ultra-low dose of quinidine. The incorporation of deuterium serves to reduce dextromethorphan first-pass liver metabolism and quinidine dosage, potentially reducing the risk of drug interactions and cardiac effects. A 10-week phase II RCT designed to evaluate the efficacy, safety, and tolerability of AVP 786 as an adjunctive therapy in patients with TRD is underway. ${ }^{51}$

\section{AZD6765 (Lanicemine)}

AZD765 is an NMDA channel blocker. Recent phase II clinical trials to evaluate whether a single infusion of AZD6765 could produce rapid antidepressant effects in subjects with TRD showed some promise. ${ }^{56}$ The primary outcome measure was the MADRS, which was used to rate overall depressive symptoms at baseline and 60, 80, 110, and 230 minutes post-infusion and on days 1, 2, 3, and 7 post-infusion. AZD6765 infusion was associated with a rapid, but short-lived improvement of antidepressant efficacy compared with placebo. No dissociative side effects were noted.

\section{Dextromethorphan}

Dextromethorphan resembles ketamine in its rapid-acting antidepressant properties including sigma- 1 and mu receptor agonist, NMDA high affinity PCP site, NMDR2A, NMDR-2B, serotonin transporter, and calcium channels antagonist, and likely activation of dendritogenesis, spinogenesis, synaptogenesis, and neuronal survival. ${ }^{57} \mathrm{~A}$ case report highlighted the role of dextromethorphan as an adjunctive agent to antidepressants resulting in rapid remission of symptoms in TRD. ${ }^{57}$ To date, no RCTs have explored dextromethorphan as monotherapy for the treatment of depressive disorders.

\section{D-Cycloserine}

D-Cycloserine is a broad spectrum antibiotic and also a partial agonist at the NMDA receptor's glycine site. At doses $\geq 100 \mathrm{mg} / \mathrm{day}$, it is a functional NMDA receptor antagonist. In an initial six-week, placebo-controlled, cross- over trial of $250 \mathrm{mg}$ /day as adjunctive treatment in TRD, it reduced depressive symptoms but did not separate from placebo due to a high placebo response rate. ${ }^{58}$ Another trial of 26 TRD patients (by the same research group and with the same trial design) assessed the efficacy of escalating the dose (up to $1000 \mathrm{mg} /$ day) ${ }^{59}$ In this trial, higher-dose of d-cycloserine showed improved antidepressant effects.

\section{Anti-Inflammatory Medications}

Activation of the peripheral immune system and inflammatory cytokines has been consistently associated with depression. ${ }^{60}$ Data indicate that inflammatory cytokines can disrupt and circumvent mechanisms of action of conventional antidepressant medications by increase in expression and activity of monoamine transporters, the primary antidepressant target for monoamine reuptake inhibition. ${ }^{60}$ Inflammation reduces availability of the enzyme co-factor, tetrahydrobiopterin, which is essential for activities of tryptophan hydroxylase and tyrosine hydroxylase, which are rate limiting enzymes for synthesis of serotonin, norepinephrine and dopamine. ${ }^{61}$ Inflammatory cytokines have also been shown to inhibit neurogenesis, through activation of nuclear factor kappa $\mathrm{B}$, which is an important component of the beneficial effects of conventional antidepressants. ${ }^{62}$

A recent report found a high correlation between plasma and cerebrospinal fluid levels of C-reactive protein (CRP), suggesting that peripheral measures of inflammation are good indicators of central nervous system inflammation. ${ }^{63}$ CRP is an acute-phase protein that is widely used in clinical practice and has also been measured in many prior studies of MDD. One study recruited 102 treatment-resistant patients experiencing depression, 48 treatment-responsive patients with MDD not currently experiencing depression, 48 patients with depression who were not receiving medication and 54 healthy volunteers. ${ }^{64}$ They found that CRP was elevated in patients with depression, and more so in treatment-resistant patients suggesting there may be a clinically and immunologically diagnosable subsyndrome of "inflamed depression" comprising the patients with MDD most likely to benefit therapeutically from treatment with anti-inflammatory drugs.

\section{Non-Steroidal Anti-Inflammatory Medications}

A meta-analysis of RCTs focusing on non-steroidal antiinflammatory drugs (NSAIDs) given as sole treatment or 
as adjunct to antidepressants, indicates that they may be more effective than placebo in treating depression. ${ }^{65}$ NSAIDs are broad-spectrum anti-inflammatory agents that also act on other targets, such as GR so the extent to which the antidepressant effect of NSAIDs is due solely to anti-inflammatory action is unclear. Four small RCTs suggest that celecoxib (200-400 mg/d) augmentation of antidepressant medication improved 4-6 week outcomes in MDD. ${ }^{66}$ There are no data, however, to support the use of celecoxib or other NSAIDs in TRD. There are also concerns about adverse events and pharmacodynamic drug interactions.

\section{Cytokine Modulators}

Cytokine modulators, including monoclonal antibodies and cytokine inhibitors, constitute a more pure antiinflammatory class because they target specific cytokine pathways. Raison and colleagues looked at whether inhibition of the inflammatory cytokine tumor necrosis factor (TNF)-alpha reduces depressive symptoms in patients with TRD and whether increased baseline plasma inflammatory biomarkers including CRP, TNF-alpha and its soluble receptors predict treatment response. ${ }^{67}$ Three infusions of infliximab (antibody directed at TNF-alpha) or placebo at baseline, weeks 2 and 6 of the 12-week trial were administered. No overall differences in improved symptoms between treatment groups across time was found. Baseline concentrations of TNF-alpha and its soluble receptors were significantly higher in infliximab-treated responders versus non-responders, and infliximab-treated responders exhibited significantly greater decreases in CRP from baseline to week 12 compared to placebotreated responders.

Another meta-analysis of RCTs looked at the role of anti-cytokine treatments (adalimumab, etanercept, infliximab and tocilizumab) in depression. ${ }^{68}$ Seven RCTs involving 2370 participants showed significant antidepressant effects of anti-cytokine treatment compared with placebo. Anti-tumour necrosis factor drugs were most commonly studied (in 5 RCTs). Separate metaanalyses of two RCTs of adjunctive treatment with anticytokine therapy and eight non-randomised and nonplacebo studies yielded similar small-to-medium effect estimates favouring anti-cytokine therapy, respectively. Adalimumab, etanercept, infliximab and tocilizumab all showed statistically significant improvements in depressive symptoms. Meta-regression exploring predictors of response found that the antidepressant effect was associated with baseline symptom severity but not with improvement in primary physical illness, sex, age or study duration. The findings indicate a potentially causal role for cytokines in depression and that cytokine modulators may be novel drugs for depression in chronically inflamed subjects with co-existing conditions of psoriasis, crohn's disease and atopic dermatitis. There is a need for RCTs of cytokine modulators using depression and TRD as the primary outcome in subjects who are free of other physical illnesses.

\section{Minocycline}

Minocycline is a tetracycline antibiotic with good CNS penetration that exerts effects on multiple interacting symptoms implicated in the pathophysiology of mood disorders. It has been used for augmentation in treatment of depression. A multi-site, 12-week, double-blind, placebo-controlled, pilot trial looked at role of adding minocycline to patients receiving treatment suffering from TRD. ${ }^{69}$ Minocycline was started at $100 \mathrm{mg}$ once daily and increased to $200 \mathrm{mg}$ after 2 weeks. 41 participants were randomized, with 21 in the minocycline group and 20 in the placebo group. A large decrease in HAMD scores was observed in the minocycline group compared to the placebo group and other scales showed moderate improvements. Findings indicated that adjunctive minocycline led to improvement in symptoms of TRD. However, the findings require replication in a larger sample.

\section{Psychedelic Agents}

Since the 1950s, multiple studies have looked at the role of psilocybin and lysergic acid diethylamide (LSD) in treatment of depression, anxiety and bipolar disorder. Psychedelic drugs act as agonists of serotonin 5-HT2A receptor, which is the key target; with partial agonism of other serotonin receptors 5-HT1A and 5-HT2B. ${ }^{70}$ Despite concerns about side effects, particularly the fear of addiction and abuse, psychedelics appear to be generally welltolerated.

\section{Psilocybin}

Psilocybin has an ancient history of medicinal use. It occurs naturally in the "psilocybe" genus of mushrooms, and is structurally related to the endogenous neurotransmitter serotonin. One RCT looked at pre- and posttreatment data with psilocybin using functional magnetic resonance imaging (fMRI) in 19 patients. ${ }^{71}$ Decreased 
depressive symptoms were observed in all 19 patients at 1-week post-treatment and $47 \%$ met criteria for response at 5 weeks. Whole-brain analyses revealed post-treatment decreases in cerebral blood flow in the temporal cortex, including the amygdala which correlated with reduced depressive symptoms. An open-label trial of psilocybin conducted by Psychedelic Research Group based in the UK looked at its role for TRD. ${ }^{72} 20$ patients with TRD received two oral doses of psilocybin (10 and $25 \mathrm{mg}, 7$ days apart) in a supportive setting. Depressive symptoms were assessed from 1 week to 6 months post-treatment. Treatment was generally well tolerated. Relatively to baseline, marked reductions in depressive symptoms were observed for the first 5 weeks post-treatment. Results remained positive at 3 and 6 months. No patients sought conventional antidepressant treatment within 5 weeks of psilocybin. Psilocybin represents a promising paradigm for unresponsive depression that warrants further research in double-blind RCTs.

\section{Ayahuasca}

Ayahuasca is a brew traditionally used for healing and spiritual purposes by indigenous populations of the Amazon Basin. A double-blind RCT involving 29 patients looked at the role of ayahuasca in TRD. ${ }^{73}$ Patients received a single dose of either ayahuasca or placebo. Changes in depression severity using scales were assessed at baseline, and at 1, 2, and 7 days after dosing. Response rates were high for both groups at day 1 and 2, and significantly higher in the ayahuasca group at day 7 . Remission rate showed a trend toward significance at day 7. No serious adverse events were observed during or after dosing. However, RCTs in larger populations are required.

\section{Lysergic Acid Diethylamide (LSD)}

LSD, often referred to by the street name "acid", is one of the most potent psychedelic agents. Unlike psilocybin, it has also been shown to affect dopaminergic neurotransmission without direct D2 receptor stimulation. While most early clinical trials used LSD, contemporary trials tend to use psilocybin due to its shorter duration of action and less controversial history, therefore, there are not as many studies with LSD compared to those on psilocybin. ${ }^{74}$ Multiple placebo-controlled studies on healthy volunteers and one study on the effect of LSD on anxiety among patients with terminal cancer have been conducted. ${ }^{74}$ Several RCTs have also been conducted to look at its role in alcohol use disorder. Currently, there is only one ongoing RCT looking at the role of LSD in depression which may extend to its role in TRD. ${ }^{74}$

\section{Medications Targeting the Dopaminergic System}

Anhedonia, a core symptom of MDD, involves downregulation of the dopaminergic system. Studies demonstrate deficits of the dopaminergic system in depression and the origin of these deficits likely lies in disruption of its regulatory afferent circuits. ${ }^{75}$ This leads to the consideration of role of dopamine agonists in treatment of MDD and TRD.

\section{Pramipexole}

Pramipexole is a dopamine agonist used for treatment of Parkinson's disease and restless leg syndrome. A 8 week double blind RCT looked at the antidepressant efficacy of a flexible dose of the dopamine agonist pramipexole as an adjunct to standard antidepressant treatment in treatment refractory depression. ${ }^{76} 40 \%$ and $33 \%$ of 60 patients randomized to augmentation with pramipexole achieved response and remission respectively, compared to $27 \%$ and $23 \%$ with placebo. Augmentation with pramipexole was well-tolerated, with no serious adverse effects identified. Another study recruited 17 patients with MDD who had failed to respond to previous treatment with a selective serotonin reuptake inhibitor. ${ }^{77} 5$ patients were diagnosed as having Bipolar 2 Disorder and 12 as having MDD. Pramipexole was added to augment medication. In total, $71 \%$ patients were responders based on the definition of $50 \%$ or more reduction in the HDRS-21 score and 59\% patients remitted. These results were almost unchanged when the sample was confined to patients with MDD.

\section{Ropinirole}

Ropinirole is another dopamine agonist with similar uses as Pramipexole. A small 16 week pilot study recruited 10 patients with TRD, out of which 7 had MDD and 3 had Bipolar 2 disorder. ${ }^{78}$ Ropinirole 0.25 to $1.5 \mathrm{mg}$ daily was added to tricyclic antidepressants or selective serotonin reuptake inhibitors and assessments were conducted at baseline and at weeks 2, 4, 8, 12, and 16. At endpoint, $40 \%$ patients were considered responders, and antidepressant augmentation was well-tolerated. 


\section{Medications Targeting the Cholinergic \\ System \\ Scopolamine}

Scopolamine is an anticholinergic agent that is used for nausea and vomiting associated with motion sickness and postoperative nausea and vomiting. Studies have shown that intravenous scopolamine has a role as a rapid-acting antidepressant for treatment of MDD. ${ }^{79}$ Scopolamine's mechanism of action is thought to stem from the convergent activation of synaptic plasticity and synaptogenesis as well as effects on glutamatergic activity occurring via antagonistic effects at muscarinic receptors. ${ }^{80}$ A study applied genome-wide markers to investigate the role of genetic variants in predicting acute antidepressant response to both ketamine and scopolamine. ${ }^{80}$ The scopolamine-treated sample comprised 37 subjects diagnosed with either MDD or BD who met criteria for TRD. Findings revealed that genetic variants associated with ketamine response accounted for $\sim 6 \%$ of the variance in scopolamine response, suggesting modest potential genetic overlap in predictors of response to these agents. More studies are need to look at role of scopolamine for sustained efficacy in TRD.

\section{Other Medications}

\section{Riluzole}

Riluzole is a member of the benzothiazole class that blocks glutamatergic neurotransmission in the CNS and is FDA approved for treatment of Amyotrophic Lateral Sclerosis. It has been studied as an adjunctive in treatment for MDD. A study analyzed 66 patients with TRD who received adjunctive riluzole in a 12-week open-label extension phase. ${ }^{81}$ Among acute phase responders, the 12 week maintained response rate was $66.7 \%$ and the relapse rate was $8.3 \%$. Longer-term tolerability of adjunctive riluzole was relatively good suggesting its potential role in TRD. More studies with larger sample sizes are needed.

\section{Neuromodulation Techniques Deep Brain Stimulation (DBS)}

DBS is an established treatment choice for Parkinson's disease, essential tremor and movement disorders, its effectiveness for the management of TRD remains unclear. An integrative review on major neuroanatomical targets of DBS was conducted for the treatment of TRD. ${ }^{82}$ Six unique DBS targets namely the subcallosal cingulate gyrus (SCG), nucleus accumbens (NAc), ventral capsule/ ventral striatum $(\mathrm{VC} / \mathrm{Vs})$ or anterior limb of internal capsule (ALIC), medial forebrain bundle (MFB), lateral habenula $(\mathrm{LHb})$ and inferior thalamic peduncle were the focus. Electrical stimulation of these brain regions particularly SCG, ALIC and MFB yielded more consistent antidepressant response rates in samples with TRD. Another study looked at the MFB as a target for DBS in MDD ${ }^{83}$ The role of MFB in depression is highlighted by the dysregulation of BDNF and neuroimmune mediators in a stress-driven chronic depression animal model. The adrenocorticotropic hormone levels and BDNF levels dropped significantly in plasma and CSF after MFB-DBS treatment indicating its potential role in future studies.

\section{Repetitive Transcranial Magnetic Stimulation (rTMS)}

Repetitive transcranial magnetic stimulation is a noninvasive procedure involving changes in cortical electrical activity induced by a rapidly-changing magnetic field which is created by a plastic-encased coil that is placed over the patient's scalp for 18-20 minutes during 30-36 sessions. ${ }^{84}$ A systematic review and meta-analysis of 73 articles (including 3 on youth) was conducted to establish the efficacy and optimal protocol for rTMS among adults and youth with TRD. ${ }^{84}$ The evidence indicates that rTMS is approximately twice as effective as a sham procedure, although the optimal rTMS protocol remains unclear, and it also appears to be a promising treatment for TRD in youth. A newer modality of rTMS is theta burst stimulation in which relatively greater amounts of stimulation can be delivered to the brain in a shorter span of time and the latest research finds this modality to be non-inferior to rTMS when used in TRD. ${ }^{85}$

\section{Vagal Nerve Stimulation (VNS)}

Food and Drugs Administration approved VNS as an adjunctive long-term treatment of TRD in $2005 .{ }^{86} \mathrm{VNS}$ procedure involves implantation of a stimulation generator connected to electrodes that are placed around the left vagus nerve under local or general anesthesia usually by a neurosurgeon. ${ }^{86}$ By electrical stimulation of the nerve, areas in brain (locus coeruleus, dorsal raphe nucleus) involved in depression are innervated, either directly or indirectly, from projections of afferent vagal fibers terminating in the nucleus tractus solitaries. In one RCT, 331 patients with TRD were divided in to 3 dose groups of stimulation low, medium and high. All groups showed statistically significant improvement in symptoms and the effect was durable over 1 year. Significantly greater durability of response was seen at medium and high doses. ${ }^{87}$ 


\section{Probiotics}

The existence of a link between the GI system and the brain has been underlined due to high co-morbidity between stress-related diseases (anxiety, depression) with gastrointestinal disorders such as irritable bowel syndrome and inflammatory bowel disorder. Three potential mechanisms have been suggested on how microbiota from the gut influences depression. ${ }^{88}$ One of them is inflammation since IgA and IgM mediated inflammatory responses to lipopolysaccharide are elevated in depressed individuals, secondly by alterations in HPA activity, and third potential mechanism is direct interference with neurotransmitter signaling. ${ }^{88}$ There are only scarce data on the use of probiotics as food and their influence on mood and GI changes. Probiotics might offer a useful novel therapeutic approach to psychiatric disorders, such as major depressive disorder although well-designed clinical trials are needed to make clear conclusions.

\section{Magnesium}

Magnesium supplementation has been linked to improvements in symptoms of major depression and postpartum depression. ${ }^{89}$ One study explored the relationship of magnesium intake and depression in 8894 US adults and found a significant association between very low magnesium intake and depression, especially in younger adults. ${ }^{89}$ The elevation of brain magnesium has been noted to be effective in preventing and reversing cognitive deficit in an Alzheimer's disease mouse model. ${ }^{90}$ Based on its role in improving cognitive functioning, more conclusive studies are needed to look at the potential role of magnesium in treating TRD.

\section{Omega-3 Polyunsaturated Fatty Acids (PUFAs)}

Supplementation with the two main types of omega-3 PUFAs, eicosapentaenoic acid (EPA) and docosahexaenoic acid (DHA), has been found to be effective in reducing symptoms of depression. ${ }^{91}$ EPA and DHA may play different roles in depression because of their involvement in anti-inflammatory activity and their maintenance of membrane integrity and fluidity, respectively. ${ }^{91}$ A meta-analysis of double-blind placebo-controlled RCTs to estimate the efficacy of EPA and DHA in the improvement of depression revealed that compared with placebo, EPA-pure and EPA-major formulations demonstrated clinical benefits whereas DHA-pure and DHA-major formulations did not exhibit such benefits on depression symptoms. ${ }^{92}$ The dosage of EPA supplementation ranged from $180 \mathrm{mg} /$ day to $4000 \mathrm{mg} /$ day. The results indicated that with an EPA dosage $\leq 1 \mathrm{~g} /$ day, the EPA-pure and EPA-major groups demonstrated significant beneficial effects on the improvement of depression as compared to $>1 \mathrm{mg}$ /day dosages. Ketogenic diet that is low in carbohydrates and high in essential PUFAs like EPA and DHA has been hypothesized to reduce anxiety and inflammation. ${ }^{93}$ The American Psychiatric Association guidelines support omega-3 consumption of at least $1 \mathrm{~g}$ of EPA and DHA daily, which is in line with the guidelines of the American Heart Association. ${ }^{93}$

\section{Exercise}

A number of studies have sought to establish whether exercise could be regarded as an alternative to antidepressants. A systematic review of studies evaluated the effectiveness of exercise as an adjunctive treatment with antidepressants on depression. ${ }^{94}$ The encompassed studies showed a strong effectiveness of exercise combined with antidepressants for MDD and TRD. There is evidence supporting the role of aerobic exercises in the treatment of depression, and evidence-based recommendations for prescribing exercises in the treatment of MDD have previously been discussed. ${ }^{95}$ No trials have described worsening in depressive symptoms associated with exercise.

\section{Yoga}

A RCT of weekly yoga classes versus health education classes in individuals with depression symptoms despite antidepressant medication use was conducted over a period of 10 weeks. ${ }^{96}$ Follow-up assessments were done 3 and 6 months afterwards. At 10 weeks, a statistically significant difference between depression symptoms among the groups was not seen. However, over the entire intervention and follow-up period, when controlling for baseline, yoga participants showed lower levels of depression than health education class participants. At 6-month follow-up, $51 \%$ of yoga participants demonstrated a response which may indicate that benefits of yoga may accumulate over time.

\section{Mindfulness-Based Meditation}

A review of studies on mindfulness-based training found it to be as effective as other behavioral therapies and pharmacologic treatments. Authors concluded that it was especially effective in reducing anxiety, depression, and stress. ${ }^{97}$ A recent systematic review and meta-analysis of 


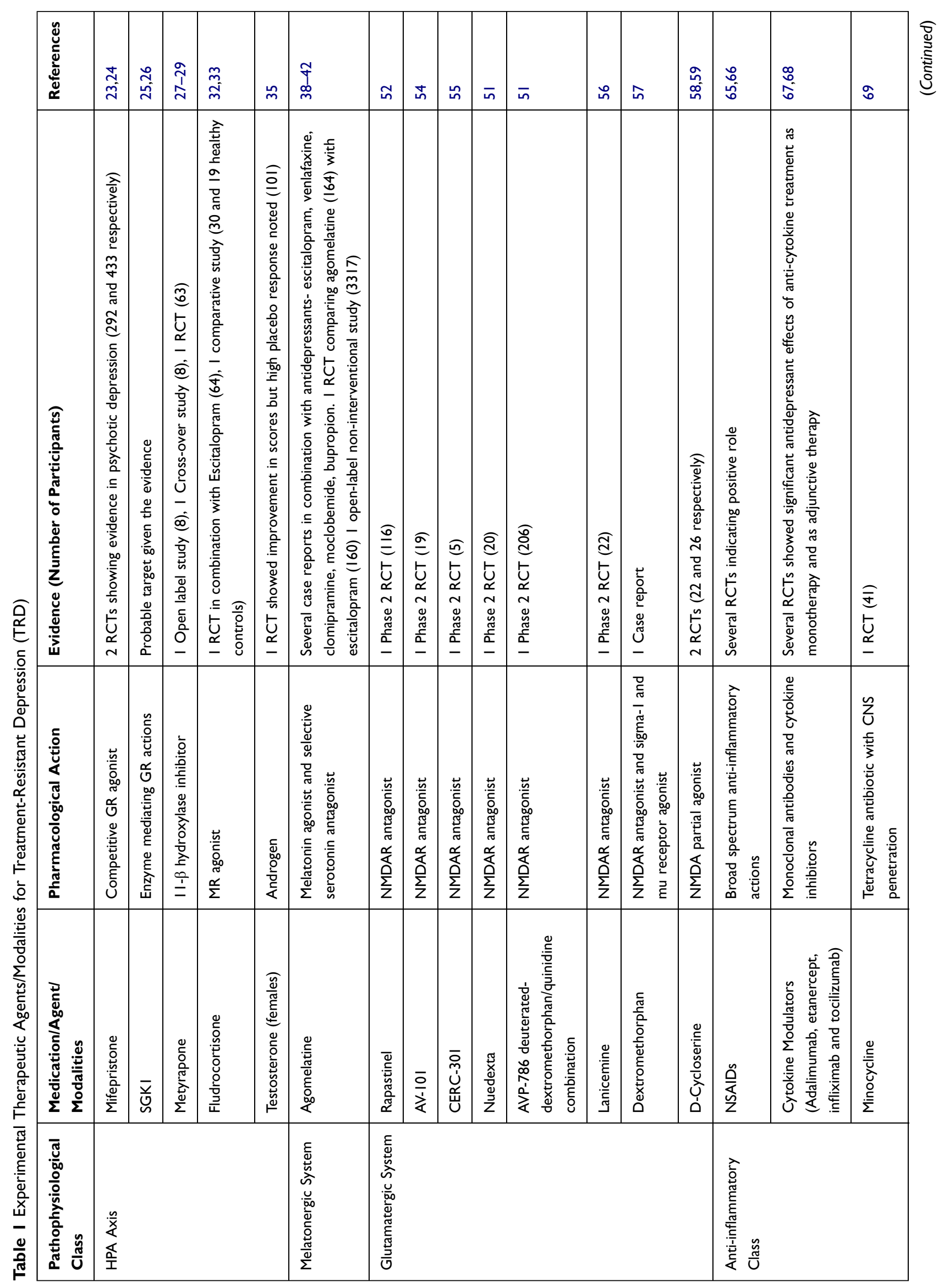




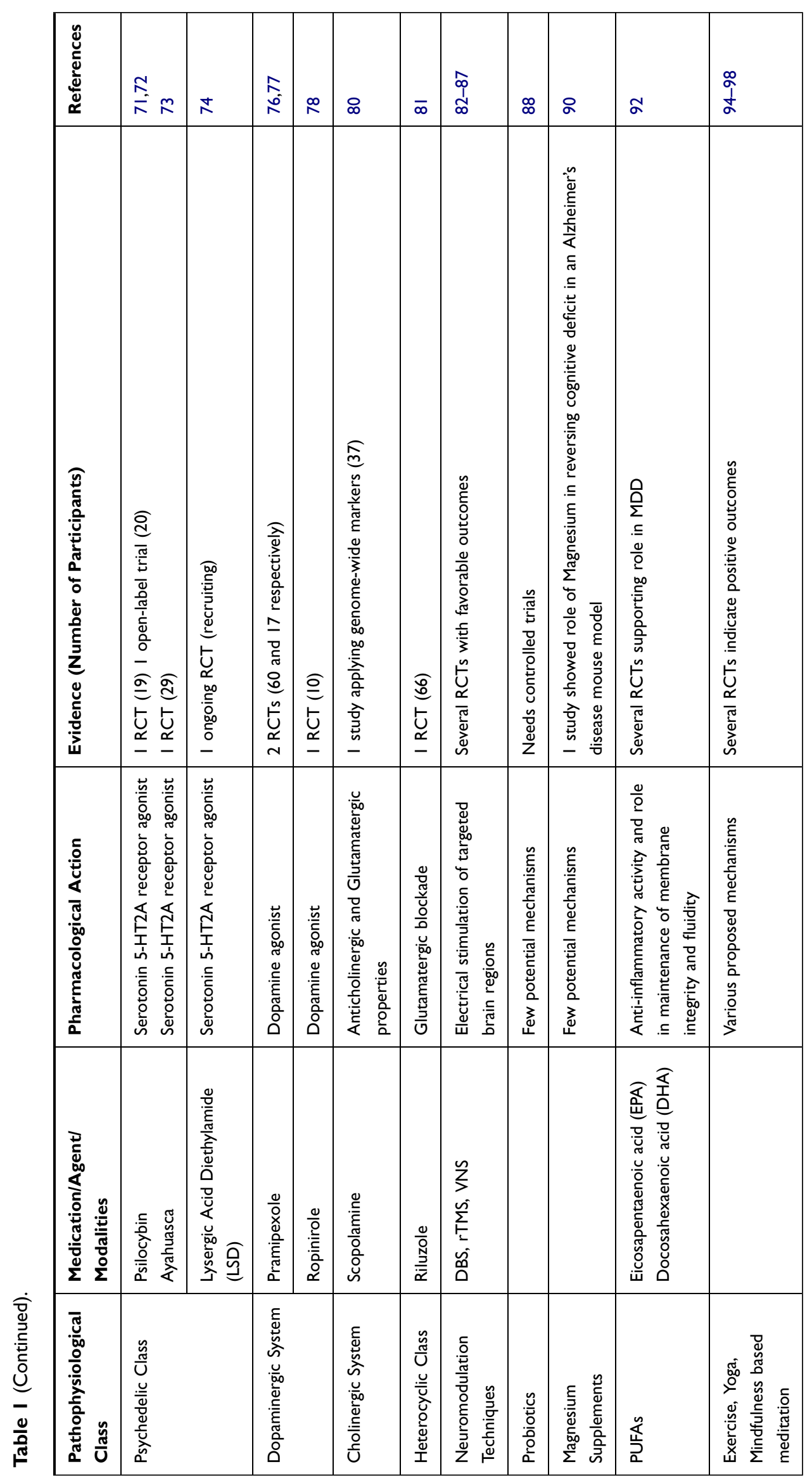


mindfulness based interventions for psychiatric disorders found the clearest evidence for their use for depression. ${ }^{98}$

\section{Conclusions}

This article reviews novel therapeutic modalities directed to manage TRD (summarized in Table 1). The proposed options are still at early stages of investigation and their efficacy in TRD remains to be established. Each option has to be viewed as a feasible option after reviewing their potential benefits versus the risks associated with their use. It is pertinent to note that, the re-evaluation to rule out other psychiatric conditions like bipolar disorder (BD) or substance use disorders remains crucial for optimal management. The role of treating co-morbid psychiatric conditions is also pertinent as treatment of co-morbidity significantly influences the symptoms of depression. The necessity to manage psychosocial factors that may be attributing to the symptoms and contributing to the lack of adequate response should not be overlooked. With TRD presenting as a challenge in clinical practice and absence of significant improvement with conventional first line medications and combinations the significance of trial of other novel treatment options is highlighted.

\section{Funding}

This research received no external funding.

\section{Disclosure}

The authors declare no conflict of interest.

\section{References}

1. GBD 2017 Disease and Injury Incidence and Prevalence Collaborators. Global, regional, and national incidence, prevalence, and years lived with disability for 354 diseases and injuries for 195 countries and territories, 1990-2017: a systematic analysis for the Global Burden of Disease Study 2017. Lancet. 2018;392 (10159):1789-1858.

2. Center for Behavioral Health Statistics and Quality. 2017 National Survey on Drug Use and Health: Methodological Summary and Definitions. Rockville, MD: Substance Abuse and Mental Health Services Administration; 2018.

3. American Psychiatric Association. Depressive disorders. In: Diagnostic and Statistical Manual of Mental Disorders. 5th ed. 2013.

4. Akil H, Gordon J, Hen R, et al. Treatment resistant depression: a multi-scale, systems biology approach. Neurosci Biobehav Rev. 2018;84:272-288. doi:10.1016/j.neubiorev.2017.08.019

5. Geschwind DH, Flint J. Genetics and genomics of psychiatric disease. Science. 2015;349:1489-1494. doi:10.1126/science.aaa8954

6. Otte C, Gold SM, Penninx BW, et al. Major depressive disorder. Nat Rev Dis Primers. 2016;2:16065. doi:10.1038/nrdp.2016.65

7. Fava GA. Can long-term treatment with antidepressant drugs worsen the course of depression? J Clin Psychiatry. 2003;64:123-133. doi:10.4088/JCP.v64n0204
8. Conway CR, George MS, Sackheim HA. Toward an evidence-based, operational definition of treatment-resistant depression. When enough is enough. JAMA Psychiatry. 2017;74:9-10. doi:10.1001/jamapsychiatry.2016.2586

9. Giakoumatos CI, Osser D. The psychopharmacology algorithm project at the harvard south shore program: an update on unipolar nonpsychotic depression. Harv Rev Psychiatry. 2019;27(1):33-52. doi:10.1097/HRP.0000000000000197

10. Anderson IM. We all know what we mean by treatment-resistant depression - don't we? Br J Psychiatry. 2018;212(5):259-261. doi:10.1192/bjp.2018.56

11. Sackeim HA. The definition and meaning of treatment-resistant depression. J Clin Psychiatry. 2001;62(Suppl 16):10-17.

12. Rush AJ, Aaronson ST, Demyttenaere K. Difficult-to-treat depression: a clinical and research roadmap for when remission is elusive. Aust N Z J Psychiatry. 2019;53(2):109-118. doi:10.1177/ 0004867418808585

13. Gaynes BN, Rush AJ, Trivedi MH, et al. The STAR*D study: treating depression in the real world. Cleve Clin J Med. 2008;75:57-66. doi:10.3949/ccjm.75.1.57

14. Williams LM, Debattista C, Duchemin AM, Schatzberg AF, Nemeroff CB. Childhood trauma predicts antidepressant response in adults with major depression: data from the randomized international study to predict optimized treatment for depression. Transl Psychiatry. 2016;6:e799. doi:10.1038/tp.2016.61

15. Salerian AJ, Altar CA. The prefrontal cortex influence over subcortical and limbic regions governs antidepressant response by $\mathrm{N}=\mathrm{H} /(\mathrm{M}+\mathrm{R}) . \quad$ Psychiatry Res. 2012;204:1-12. doi:10.1016/j. pscychresns.2012.04.019

16. Greicius MD, Flores $\mathrm{BH}$, Menon V, et al. Resting-state functional connectivity in major depression: abnormally increased contributions from subgenual cingulate cortex and thalamus. Biol Psychiatry. 2007;62:429-437. doi:10.1016/j.biopsych.2006.09.020

17. Thase ME, Schwartz TL. Choosing medications for treatment-resistant depression based on mechanism of action. J Clin Psychiatry. 2015;76:720-727. doi:10.4088/JCP.14052ah2c

18. Denton WH, Carmody TJ, Rush AJ, et al. Dyadic discord at baseline is associated with lack of remission in the acute treatment of chronic depression. Psychol Med. 2010;40:415-424. doi:10.1017/ S0033291709990535

19. Caraci F, Calabrese F, Molteni R, et al. International union of basic and clinical pharmacology CIV: the neurobiology of treatment-resistant depression: from antidepressant classifications to novel pharmacological targets. Pharmacol Rev. 2018;70(3):475-504. doi:10.1124/pr.117.014977

20. Nikkheslat N, McLaughlin AP, Hastings C, et al. Childhood trauma, HPA axis activity and antidepressant response in patients with depression. Brain Behav Immun. 2020;87:229-237. doi:10.1016/j. bbi.2019.11.024

21. Perrin AJ, Horowitz MA, Roelofs J, Zunszain PA, Pariante CM. Glucocorticoid resistance: is it a requisite for increased cytokine production in depression? A systematic review and meta-analysis. Front Psychiatry. 2019;10:423. doi:10.3389/fpsyt.2019.00423

22. Flores BH, Kenna H, Keller J, Solvason HB, Schatzberg AF. Clinical and biological effects of mifepristone treatment for psychotic depression. Neuropsychopharmacology. 2006;31(3):628-636. doi:10.1038/sj.npp.1300884

23. Block T, Petrides G, Kushner H, Kalin N, Belanoff J, Schatzberg A. Mifepristone plasma level and glucocorticoid receptor antagonism associated with response in patients with psychotic depression. J Clin Psychopharmacol. 2017;37(5):505-511. doi:10.1097/ JCP.0000000000000744

24. Blasey CM, Block TS, Belanoff JK, Roe RL. Efficacy and safety of mifepristone for the treatment of psychotic depression. J Clin Psychopharmacol. 2011;31(4):436-440. doi:10.1097/ JCP.0b013e3182239191 
25. Yuen EY, Liu W, Karatsoreos IN, et al. Mechanisms for acute stress-induced enhancement of glutamatergic transmission and working memory. Mol Psychiatry. 2011;16(2):156-170. doi:10.1038/mp.2010.50

26. Anacker C, Cattaneo A, Musaelyan K, et al. Role for the kinase SGK1 in stress, depression, and glucocorticoid effects on hippocampal neurogenesis. Proc Natl Acad Sci U $S$ A. 2013;110 (21):8708-8713. doi:10.1073/pnas.1300886110

27. Murphy BE, Dhar V, Ghadirian AM, Chouinard G, Keller R. Response to steroid suppression in major depression resistant to antidepressant therapy. J Clin Psychopharmacol. 1991;11:121-126.

28. O'Dwyer AM, Lightman SL, Marks MN, Checkley SA. Treatment of major depression with metyrapone and hydrocortisone. J Affect Disord. 1995;33:123-128. doi:10.1016/0165-0327(94)00082-K

29. Jahn H, Schick M, Kiefer F, Kellner M, Yassouridis A, Wiedemann K. Metyrapone as additive treatment in major depression: a double-blind and placebo-controlled trial. Arch Gen Psychiatry. 2004;61:1235-1244. doi:10.1001/archpsyc.61.12.1235

30. de Kloet ER, Joels M, et al. Stress and the brain: from adaptation to disease. Nature Rev. 2005;6:6. doi:10.1038/nrn1683

31. Yau JL, Hibberd C, et al. The effect of chronic fluoxetine treatment on brain corticosteroid receptor mRNA expression and spatial memory in young and aged rats. Brain Res Mol Brain Res. 2002;106:117-123. doi:10.1016/S0169-328X(02)00418-7

32. Otte C, Hinkelmann $\mathrm{K}$, et al. Modulation of the mineralocorticoid receptor as add-on treatment in depression: a randomized, double-blind, placebo-controlled proof-of-concept study. J Psychiatr Res. 2010;44(6):339-346. doi:10.1016/j.jpsychires.2009.10.006

33. Lembke A, Gomez R, Tenakoon L, et al. The mineralocorticoid receptor agonist, fludrocortisone, differentially inhibits pituitary-adrenal activity in humans with psychotic major depression. Psychoneuroendocrinology. 2013;38(1):115-121. doi:10.1016/j.psyneuen.2012.05.006

34. US Food and Drug Administration: FDA approved first treatment for post-partum depression. FDA news release; March 19, 2019. Available from: https://www.fda.gov/news-events/pressannouncements/fda-approves-first-treatment-post-partum-depression. Accessed January 27, 2021.

35. Dichtel LE, Carpenter LL, Nyer M, et al. Low dose Testosterone Augmentation for Antidepressant-Resistant Major Depressive Disorder in Women: an 8-week Randomized Placebo-controlled Study. Am J Psychiatry. 2020;177:965-973. doi:10.1176/appi.ajp.2020.19080844

36. Liu J, Clough SJ, Dubocovich ML. Role of the MT1 and MT2 melatonin receptors in mediating depressive- and anxiety-like behaviors in $\mathrm{C} 3 \mathrm{H} / \mathrm{HeN}$ mice. Genes Brain Behav. 2017;16:546-553. doi:10.1111/gbb.12369

37. Valdés-Tovar M, Estrada-Reyes R, Solís-Chagoyán H, et al. Circadian modulation of neuroplasticity by melatonin: a target in the treatment of depression. Br J Pharmacol. 2018;175(16):3200-3208. doi:10.1111/ bph.14197

38. Potměšil P. What combinations of agomelatine with other antidepressants could be successful during the treatment of major depressive disorder or anxiety disorders in clinical practice? Ther Adv Psychopharmacol. 2019;9:2045125319855206. doi:10.1177/2045125319855206

39. Stuhec M, Oravecz R. Moclobemide as add-on therapy to agomelatine in a patient with treatment-resistant major depressive disorder: a psychopharmacological case. Wien Klin Wochenschr. 2016;128:295-298. doi:10.1007/s00508-015-0861-0

40. Suhs KW, Correll C, Eberlein CK, et al. Combination of agomelatine and bupropion for treatment-resistant depression: results from a chart review study including a matched control group. Brain Behav. 2015;5:e00318. doi:10.1002/brb3.318

41. Corruble E, de Bodinat C, Belaïdi C, Goodwin GM, agomelatine study group. Efficacy of agomelatine and escitalopram on depression, subjective sleep and emotional experiences in patients with major depressive disorder: a 24-wk randomized, controlled, double-blind trial. Int $J$ Neuropsychopharmacol. 2013;16(10):2219-2234. doi:10.1017/S1461145713000679
42. Laux G, Huttner NA; VIVALDI study group. Subgroup analysis of the non-interventional study VIVALDI: agomelatine in treatment-naive patients, in combination therapy and after treatment switch. Int J Psychiatry Clin Pract. 2014;18:86-96. doi:10.3109/ 13651501.2014.892138

43. Fatouh AM, Elshafeey AH, Abdelbary A. Intranasal agomelatine solid lipid nanoparticles to enhance brain delivery: formulation, optimization and in vivo pharmacokinetics. Drug Des Devel Ther. 2017;11:1815-1825. doi:10.2147/DDDT.S102500

44. Szarmach J, Cubała WJ, Włodarczyk A, Wiglusz MS. Short-term ketamine administration in treatment-resistant depression: focus on cardiovascular safety. Psychiatr Danub. 2019;31(Suppl 3):585-590.

45. Włodarczyk A, Cubała WJ, Szarmach J, Małyszko A, Wiglusz MS. Short-term ketamine administration in treatment-resistant depression patients: focus on adverse effects on the central nervous system. Psychiatr Danub. 2019;31(Suppl 3):530-533.

46. Singh JB, Fedgchin M, Daly EJ, et al. A double-blind, randomized, placebo-controlled, dose-frequency study of intravenous ketamine in patients with treatment-resistant depression. Am $J$ Psychiatry. 2016;173(8):816-826.

47. Daly EJ, Singh JB, Fedgchin M, et al. Efficacy and safety of intranasal esketamine adjunctive to oral antidepressant therapy in treatment-resistant depression: a randomized clinical trial. JAMA Psychiatry. 2018;75:139-148. doi:10.1001/ jamapsychiatry.2017.3739

48. Zheng W, Cai DB, Xiang YQ, et al. Adjunctive intranasal esketamine for major depressive disorder: a systematic review of randomized double-blind controlled-placebo studies. $J$ Affect Disord. 2020;265:63-70. doi:10.1016/j.jad.2020.01.002

49. Papakostas GI, Salloum NC, Hock RS, et al. Efficacy of esketamine augmentation in major depressive disorder: a meta-analysis. J Clin Psychiatry. 2020;81(4):19r12889. doi:10.4088/JCP.19r12889

50. EMA 2019. Spravato ${ }^{\circledR}$ (esketamine) summary of product characteristics. Available from: https://www.ema.europa.eu/en/documents/productinformation/spravato-epar-product-information.en.pdf. Accessed Jan 02, 2021.

51. Garay RP, Zarate CA Jr, Charpeaud T, et al. Investigational drugs in recent clinical trials for treatment-resistant depression. Expert Rev Neurother. 2017;17(6):593-609. doi:10.1080/ 14737175.2017.1283217

52. Moskal JR, Burgdorf JS, Stanton PK, et al. The Development of Rapastinel (Formerly GLYX-13); a rapid acting and long lasting antidepressant. Curr Neuropharmacol. 2016;15:47-56. doi:10.2174/ 1570159X14666160321122703

53. Savitz J. The kynurenine pathway: a finger in every pie. Mol Psychiatry. 2020;25(1):131-147. doi:10.1038/s41380-019-0414-4

54. Park LT, Kadriu B, Gould TD, et al. A randomized trial of the N-methyl-d-aspartate receptor glycine site antagonist prodrug 4-chlorokynurenine in treatment-resistant depression. Int $J$ Neuropsychopharmacol. 2020;23(7):417-425. doi:10.1093/ ijnp/pyaa025

55. Ibrahim L, Diaz Granados N, Jolkovsky L, et al. A Randomized, placebo-controlled, crossover pilot trial of the oral selective NR2B antagonist MK-0657 in patients with treatment-resistant major depressive disorder. J Clin Psychopharmacol. 2012;32:551-557. doi:10.1097/JCP.0b013e31825d70d6

56. Zarate CA Jr, Mathews D, Ibrahim L, et al. A randomized trial of a low-trapping nonselective N-methyl-D-aspartate channel blocker in major depression. Biol Psychiatry. 2013;74:257-264. doi:10.1016/j. biopsych.2012.10.019

57. Lauterbach EC. Treatment resistant depression with loss of antidepressant response: rapid-acting antidepressant action of dextromethorphan, a possible treatment bridging molecule. Psychopharmacol Bull. 2016;46(2):53-58. 
58. Heresco-Levy U, Javitt DC, Gelfin Y, et al. Controlled trial of D-cycloserine adjuvant therapy for treatment-resistant major depressive disorder. J Affect Disord. 2006;93(1-3):239-243. doi:10.1016/j. jad.2006.03.004

59. Heresco-Levy U, Gelfin G, Bloch B, et al. A randomized add-on trial of high-dose D-cycloserine for treatment-resistant depression. Int J Neuropsychopharmacol. 2013;16(3):501-506. doi:10.1017/ S1461145712000910

60. Zhu CB, Lindler KM, Owens AW, Daws LC, Blakely RD, Hewlett WA. Interleukin-1 receptor activation by systemic lipopolysaccharide induces behavioral despair linked to MAPK regulation of CNS serotonin transporters. Neuropsychopharmacology. 2010;35:2510-2520. doi:10.1038/npp.2010.116

61. Haroon E, Raison CL, Miller AH. Psychoneuroimmunology meets neuropsychopharmacology: translational implications of the impact of inflammation on behavior. Neuropsychopharmacology. 2012;37 (1):137-162.

62. Koo JW, Russo SJ, Ferguson D, Nestler EJ, Duman RS. Nuclear factor-kappaB is a critical mediator of stress-impaired neurogenesis and depressive behavior. Proc Natl Acad Sci U S A. 2010;107 (6):2669-2674. doi:10.1073/pnas.0910658107

63. Felger JC, Haroon E, Patel TA, et al. What does plasma CRP tell us about peripheral and central inflammation in depression? Mol Psychiatry. 2020;25(6):1301-1311. doi:10.1038/s41380-018-0096-3

64. Chamberlain SR, Cavanagh J, de Boer P, et al. Treatment-resistant depression and peripheral C-reactive protein. $\mathrm{Br} J$ Psychiatry. 2019;214(1):11-19. doi:10.1192/bjp.2018.66

65. Kohler O, Benros ME, Nordentoft M, et al. Effect of anti-inflammatory treatment on depression, depressive symptoms, and adverse effects: a systematic review and meta-analysis of randomized clinical trials. JAMA Psychiatry. 2014;71:1381-1391. doi:10.1001/jamapsychiatry.2014.1611

66. Andrade C. Antidepressant augmentation with anti-inflammatory agents. J Clin Psychiatry. 2014;75(9):975-977. doi:10.4088/ JCP.14f09432

67. Raison CL, Rutherford RE, Woolwine BJ, et al. A randomized controlled trial of the tumor necrosis factor antagonist infliximab for treatment-resistant depression: the role of baseline inflammatory biomarkers. JAMA Psychiatry. 2013;70(1):31-41. doi:10.1001/2013. jamapsychiatry.4

68. Kappelmann N, Lewis G, Dantzer R, Jones PB, Khandaker GM. Antidepressant activity of anti-cytokine treatment: a systematic review and meta-analysis of clinical trials of chronic inflammatory conditions. Mol Psychiatry. 2018;23(2):335-343. doi:10.1038/ mp.2016.167

69. Husain MI, Chaudhry IB, Husain N, et al. Minocycline as an adjunct for treatment-resistant depressive symptoms: a pilot randomised placebo-controlled trial. J Psychopharmacol. 2017;31(9):1166-1175. doi:10.1177/0269881117724352

70. Nutt D. Psychedelic drugs-a new era in psychiatry? Dialogues Clin Neurosci. 2019;21(2):139-147.

71. Carhart-Harris RL, Roseman L, Bolstridge M, et al. Psilocybin for treatment-resistant depression: fMRI-measured brain mechanisms. Sci Rep. 2017;7(1):13187. doi:10.1038/s41598-017-13282-7

72. Carhart-Harris RL, Bolstridge M, Day CMJ, et al. Psilocybin with psychological support for treatment-resistant depression: six-month follow-up. Psychopharmacology. 2018;235(2):399-408. doi:10.1007/ s00213-017-4771-x

73. Palhano-Fontes F, Barreto D, Onias H, et al. Rapid antidepressant effects of the psychedelic ayahuasca in treatment-resistant depression: a randomized placebo-controlled trial. Psychol Med. 2019;49 (4):655-663. doi:10.1017/S0033291718001356

74. Chi T, Gold JA. A review of emerging therapeutic potential of psychedelic drugs in the treatment of psychiatric illnesses. $J$ Neurol Sci. 2020;411:116715. doi:10.1016/j.jns.2020.116715
75. Belujon P, Grace AA. Dopamine system dysregulation in major depressive disorders. Int $J$ Neuropsychopharmacol. 2017;20 (12):1036-1046. doi:10.1093/ijnp/pyx056

76. Cusin C, Iovieno N, Iosifescu DV, et al. A randomized, double-blind, placebo-controlled trial of pramipexole augmentation in treatment-resistant major depressive disorder. J Clin Psychiatry. 2013;74(7):e636-41. doi:10.4088/JCP.12m08093

77. Hori H, Kunugi H. The efficacy of pramipexole, a dopamine receptor agonist, as an adjunctive treatment in treatment-resistant depression: an open-label trial. ScientificWorldJournal. 2012;2012:372474. doi: $10.1100 / 2012 / 372474$

78. Cassano P, Lattanzi L, Fava M, et al. Ropinirole in treatment-resistant depression: a 16-week pilot study. Can J Psychiatry. 2005;50 (6):357-360. doi:10.1177/070674370505000612

79. Drevets WC, Furey ML. Replication of scopolamine's antidepressant efficacy in major depressive disorder: a randomized, placebocontrolled clinical trial. Biol Psychiatry. 2010;67:432-438. doi:10.1016/j.biopsych.2009.11.021

80. Guo W, Machado-Vieira R, Mathew S, et al. Exploratory genome-wide association analysis of response to ketamine and a polygenic analysis of response to scopolamine in depression. Transl Psychiatry. 2018;8(1):280. doi:10.1038/s41398-018-0311-7

81. Sakurai H, Dording C, Yeung A, et al. Longer-term open-label study of adjunctive riluzole in treatment-resistant depression. $J$ Affect Disord. 2019;258:102-108. doi:10.1016/j.jad.2019.06.065

82. Dandekar MP, Fenoy AJ, Carvalho AF, Soares JC, Quevedo J. Deep brain stimulation for treatment-resistant depression: an integrative review of preclinical and clinical findings and translational implications. $\mathrm{Mol}$ Psychiatry. 2018;23(5):1094-1112. doi:10.1038/mp.2018.2

83. Dandekar MP, Saxena A, Scaini G, et al. Medial forebrain bundle deep brain stimulation reverses anhedonic-like behavior in a chronic model of depression: importance of BDNF and inflammatory cytokines. Mol Neurobiol. 2019;56(6):4364-4380. doi:10.1007/ s12035-018-1381-5

84. Leggett LE, Soril LJ, Coward S, Lorenzetti DL, MacKean G, Clement FM. Repetitive transcranial magnetic stimulation for treatment-resistant depression in adult and youth populations: a systematic literature review and meta-analysis. Prim Care Companion CNS Disord. 2015;17(6). doi:10.4088/PCC.15r01807

85. Somani A, Kar SK. Efficacy of repetitive transcranial magnetic stimulation in treatment-resistant depression: the evidence thus far. Gen Psychiatr. 2019;32(4):e100074. doi:10.1136/gpsych-2019-100074

86. Carreno FR, Frazer A. Vagal nerve stimulation for treatment-resistant depression. Neurotherapeutics. 2017;14(3):716-727. doi:10.1007/ s13311-017-0537-8

87. Aaronson ST, Carpenter LL, Conway CR, et al. Vagus nerve stimulation therapy randomized to different amounts of electrical charge for treatment-resistant depression: acute and chronic effects. Brain Stimul. 2013;6(4):631-640. doi:10.1016/j.brs.2012.09.013

88. Vlainić JV, Šuran J, Vlainić T, Vukorep AL. Probiotics as an adjuvant therapy in major depressive disorder. Curr Neuropharmacol. 2016;14 (8):952-958. doi:10.2174/1570159X14666160526120928

89. Tarleton EK, Littenberg B. Magnesium intake and depression in adults. J Am Board Fam Med. 2015;28:249-256. doi:10.3122/ jabfm.2015.02.140176

90. Whittle N, Li L, Chen W-Q, et al. Changes in brain protein expression are linked to magnesium restriction-induced depression-like behavior. Amino Acids. 2011;40:1231-1248. doi:10.1007/s00726010-0758-1

91. Deacon G, Kettle C, Hayes D, Dennis C, Tucci J. Omega 3 polyunsaturated fatty acids and the treatment of depression. Crit Rev Food Sci Nutr. 2017;57(1):212-223. doi:10.1080/ 10408398.2013.876959

92. Liao Y, Xie B, Zhang $\mathrm{H}$, et al. Efficacy of omega-3 PUFAs in depression: a meta-analysis. Transl Psychiatry. 2019;9(1):190. doi:10.1038/s41398-019-0515-5 
93. Włodarczyk A, Cubała WJ, Wielewicka A. Ketogenic diet: a dietary modification as an anxiolytic approach? Nutrients. 2020;12(12):3822. doi:10.3390/nu12123822

94. Mura G, Moro MF, Patten SB, Carta MG. Exercise as an add-on strategy for the treatment of major depressive disorder: a systematic review. CNS Spectr. 2014;19(6):496-508. doi:10.1017/ S1092852913000953

95. Rethorst CD, Trivedi MH. Evidence-based recommendations for the prescription of exercise for major depressive disorder. J Psychiatr Pract. 2013;19(3):204-212. doi:10.1097/01.pra.0000430504. $16952.3 \mathrm{e}$

96. Uebelacker LA, Tremont G, Gillette LT, et al. Adjunctive yoga v. health education for persistent major depression: a randomized controlled trial. Psychol Med. 2017;47(12):2130-2142. doi:10.1017/ S0033291717000575
97. Saeed SA, Cunningham K, Bloch RM. Depression and anxiety disorders: benefits of exercise, yoga, and meditation. Am Fam Physician. 2019;99(10):620-627.

98. Goldberg SB, Tucker RP, Greene PA, et al. Mindfulness-based interventions for psychiatric disorders: a systematic review and meta-analysis. Clin Psychol Rev. 2018;59:52-60. doi:10.1016/j. cpr.2017.10.011

\section{Publish your work in this journal}

The Journal of Experimental Pharmacology is an international, peerreviewed, open access journal publishing original research, reports, reviews and commentaries on all areas of laboratory and experimental pharmacology. The manuscript management system is completely online and includes a very quick and fair peer-review system. Visit http://www.dovepress.com/testimonials.php to read real quotes from published authors. 